\title{
Activating transcription factor-3 (ATF3) functions as a tumor suppressor in colon cancer and is up-regulated upon heat-shock protein 90 (Hsp90) inhibition
}

Christina Hackl ${ }^{1}$, Sven A Lang ${ }^{1}$, Christian Moser ${ }^{1}$, Akira Mori ${ }^{1}$, Stefan Fichtner-Feigl ${ }^{1}$, Claus Hellerbrand ${ }^{2}$, Wolfgang Dietmeier ${ }^{3}$, Hans J Schlitt', Edward K Geissler ${ }^{1}$, Oliver Stoeltzing ${ }^{1,4^{*}}$

\begin{abstract}
Background: Activating transcription factor-3 (ATF3) is involved in the complex process of cellular stress response. However, its exact role in cancer is discussed controversially because both tumor suppressive and oncogenic effects have been described. Here we followed-up on our previous observation that inhibition of Hsp90 may increase ATF3 expression and sought to determine the role of ATF3 in colon cancer.

Methods: Regulation of ATF3 was determined in cancer cells using signaling inhibitors and a heat-shock protein90 (Hsp90) antagonist. Human HCT116 cancer cells were stably transfected with an ATF3-shRNA or a luciferaseshRNA expression plasmid and alterations in cell motility were assessed in migration assays. The impact of ATF3 down-regulation on cancer growth and metastasis were investigated in a subcutaneous tumor model, a model of hepatic tumor growth and in a model of peritoneal carcinomatosis. Human colon cancer tissues were analyzed for ATF3 expression.

Results: The results show that therapeutic Hsp90 inhibition substantially up-regulates the expression of ATF3 in various cancer cells, including colon, gastric and pancreatic cancer. This effect was evident both in vitro and in vivo. RNAi mediated knock-down of ATF3 in HCT116 colon cancer cells significantly increased cancer cell migration in vitro. Moreover, in xenogenic mouse models, ATF3 knock-down promoted subcutaneous tumor growth and hepatic metastasis, as well as peritoneal carcinomatosis. Importantly, ATF3 expression was lower in human colon cancer specimens, as compared to corresponding normal surrounding tissues, suggesting that ATF3 may represent a down-regulated tumor suppressor in colon cancer.

Conclusion: In conclusion, ATF3 down-regulation in colon cancer promotes tumor growth and metastasis. Considering that blocking Hsp90 induces ATF3 expression, Hsp90 inhibition may represent a valid strategy to treat metastatic colon cancer by up-regulating this anti-metastatic transcription factor.
\end{abstract}

\section{Background}

Heat shock protein 90 (Hsp90) targeting has emerged as a valuable strategy for cancer therapy $[1,2]$, because these proteins are being up-regulated in malignant and non-malignant cells types upon exposure to a variety of stressors [3]. At constitutive levels, heat-shock proteins

\footnotetext{
* Correspondence: o.stoeltzing@uke.de

'Department of Surgery, University of Regensburg Medical Center, FranzJosef Strauß Allee 11, 93042 Regensburg, Germany

Full list of author information is available at the end of the article
}

regulate proper folding and stabilization of abundant intracellular proteins, and their stress-associated induction improves cell survival. Hsp90, one of the most studied molecular chaperons, is overexpressed in tumor cells and is essential for the stability and function of a wide range of oncogenic client proteins [4]. These Hsp90 clients comprise kinases such as ERBB2, EGFR, CDK4, RAF, AKT, cMET and BCR-ABL, and transcription factors such as HIF- $1 \alpha$, STAT3, and STAT5 $[2,5,6]$. Thus, Hsp90 is a promising target for cancer therapy, as
C Biomed Central 
demonstrated by the expanding armamentarium of Hsp90 inhibitors and by new clinical studies incorporating the use of these inhibitors [7]. Nevertheless, due to the broad and complex inhibition of multiple signaling pathways affected by Hsp90, the biological effects remain poorly defined and incompletely understood.

We recently demonstrated that therapeutic inhibition of Hsp90 not only elicits antineoplastic efficacy through blocking oncogenic signaling, but also up-regulates certain signaling molecules in human colon carcinoma cell lines. One of these molecules is activating transcription factor-3 (ATF3), which is Hsp90-inhibitor inducible in HCT116, SW620 and HT29 colon cancer cells [8]. Importantly, such protein up-regulation in response to Hsp90 inhibition has thus far only been reported for certain other heat-shock proteins such as HSF1 and Hsp70. This response may counteract the anti-neoplastic potential of Hsp90 inhibitors for the following reasons $[9,10]$. ATF3 belongs to the ATF/cyclic AMP response element binding (CREB) family of transcription factors and most cells have very weak or absent ATF3 expression under steady-state conditions. A significant increase in ATF3 can be observed when cell-stress is induced [11], making ATF3 an universal "adaptive response gene" $[12,13]$.

Importantly, different roles for ATF3 have been proposed. In normal tissues, ATF3 may promote both apoptosis and cell proliferation [13], while in neoplasms it has been identified as either an oncogene or as tumor suppressor, depending on tumor entity and grade [13-15]. For instance, ATF3 can mediate pro-apoptotic effects in human mammary epithelial cells, whereas in breast cancer cells (MCF10A) it may promote cell survival, motility and invasiveness [15]. Transgenic mice that overexpress ATF3 in basal epithelial cells develop epidermal hyperplasia, dysplastic lesions and oral squamous cell carcinoma [16]. Also in favor of oncogenicity, the tumor suppressor gene Drg-1 mediates its anti-metastatic properties through ATF3 down-regulation in prostate cancer [17].

In colon cancer, the effects of ATF3 expression are particularly perplexing. In one respect, ATF3 was shown to be overexpressed in human colon cancer specimens and appears to promote tumor growth and migration in an experimental HT29 colon cancer model $[18,19]$. In another respect, ATF3 has been described to mediate anti-neoplastic and anti-invasive effects of non-steroidal anti-inflammatory drugs (i.e. COX-2 inhibitors) in colorectal cancer [14]. In the present study, we sought to clarify ATF3 regulation and its role in human colon cancer using xenogenic mouse models. We hypothesized that Hsp90 inhibitor-mediated induction of ATF3 expression does not counteract the anti-neoplastic and anti-metastatic potential of Hsp90 targeting agents.

\section{Methods}

\section{Cell culture}

The human colorectal cancer cell lines HCT116, SW620 and HT29 were obtained from the American Type Culture Collection (Manassas, VA). The human gastric cancer cell line TMK-1 was obtained from Eiichi Tahara (University of Hiroshima, Hiroshima, Japan). The metastatic human pancreatic cancer cell line L3.6pl was kindly provided by Dr. I.J. Fidler (The University of Texas, MD Anderson Cancer Center, Houston, TX). HCT116 and SW620 cells were cultured in RPMI 1640, whereas TMK1, HT29 and L3.6pl were grown in DMEM supplemented with 20\% FCS (HCT116 and SW620), 15\% FCS (L3.6pl), or $10 \%$ FCS (HT29, TMK-1). All in vitro experiments were performed at $60-70 \%$ cell density to reduce effects of confluence. Cell growth rates of transfected cells were assessed by MTT assays, as previously described [20].

\section{Stable transfection}

HCT116 cells were stable transfected with either an ATF3-shRNA (TIB Molbiol; Berlin, Germany) or a luciferase-shRNA (Luc-shRNA) expression plasmid (TIB Molbiol; Berlin, Germany) by using the Lipofectamine transfection reagent (Invitrogen; Karlsruhe, Germany). Cells were grown and expanded in selective medium containing neomycin (G418, Sigma Aldrich, Deisenhofen, Germany). Successful transfection was verified by Western blotting and semi-quantitative PCR for ATF3.

\section{Reagents and antibodies}

The water-soluble Hsp90 inhibitor 17-(dimethylaminoethylamino)-17-demethoxy-geldanamycin (17-DMAG) was purchased from Invivogen (Cayla-Invivogen) and was applied as previously published [8]. Antibodies against ATF3 and anti- $\beta$-actin were obtained from Santa Cruz Biotechnology (Santa Cruz, CA). $\beta$-actin served as a loading control in Western blotting.

\section{Western blot analysis}

Protein was extracted from whole-cell lysates with RIPA buffer as described before and $50-\mu \mathrm{g}$ protein samples were subjected to Western blotting on a denaturing $10 \%$ sodium dodecyl sulfate-polyacrylamide gel [20]. Membranes were probed for ATF3 and $\beta$-actin. For induction of ATF3 in vitro, the Hsp90 inhibitor 17-DMAG (100 $\mathrm{ng} / \mathrm{ml})$ was added to cell cultures for indicated times and ATF3 protein analysis was performed thereafter. Expression of ATF3 in 17-DMAG treated tumors was similarly determined by lysis of snap frozen tumor tissues and subsequent Western blotting, as described [20].

\section{Real-time PCR}

Real-time PCR was performed as we have previously described [21]. Primer pairs were as follows: ATF3 
forward 5-'ctgcagaaagagtcggag-3' and reverse 5'tgagcccggacaatacac-3'; VEGF-A forward 5'-gcacccatggcagaaggaggag-3' and reverse 5'-agcccccgcatcgcatcag-3'; HIF- $1 \alpha$ forward taccatgcccagattcaggat and reverse tcagtggtggcagtggtagtgg; GLUT-1 forward 5'-aactcttcagccagggtccac-3' and reverse 5'-cacagtgaagatgatgaagac-3'. Real-time PCR was done using the LightCycler system and Roche fast-Start Light Cycler-Master Hybridization Probes master mix (Roche Diagnostics) [20].

\section{Migration Assays}

Migration assays were performed using modified Boyden chambers, as described elsewhere [21]. Briefly, $10^{5}$ cells were resuspended in 1\% FCS medium and seeded into $8-\mu \mathrm{m}$ filter pores inserts (Becton Dickinson Bioscience). $10 \%$ FCS-enriched medium \pm 17-DMAG (100 nM) served as chemoattractant. After incubation, migrated cells were stained (Diff-Quick reagent, Dade Behring) and counted in four random fields.

\section{Animal models}

Eight-week-old male nude mice (Charles River, Sulzfeld, Germany) were used. Experiments were approved by the Institutional Animal Care and Use Committee of the University of Regensburg and the regional authorities (Regierung der Oberpfalz, reference number HIF1_2004) and in accordance to the "Guidelines for the Welfare of Animals in Experimental Neoplasia" published by The United Kingdom Coordinating Committee on Cancer Research. In experiments, animals were weighed daily and monitored for weight-loss and other signs of distress.

\section{Tumour models}

(1) One-million human cancer cells (TMK-1, L3.6pl) were implanted into the subcutis of nude mice, as described [8]. After implantation, tumors were allowed to grow to a volume of $400 \mathrm{~mm}^{3}$ until treatment with either the Hsp90 inhibitor 17-DMAG $(3 \times 25 \mathrm{mg} / \mathrm{kg} /$ week; i.p.), or PBS (control) was started. This dose has proven antineoplastic potential in previous models $[8,20]$. Tumors were harvested after 14 days of treatment to determine ATF3 protein expression ( $n=3$ per group).

(2) One-million ATF3-shRNA, or Luc-shRNA transfected HCT116 human colorectal cancer cells were injected into the subcutis of nude mice $(n=9-10$ per group). Tumor diameters were measured every other day, and volumes calculated using the estimation: width $^{2}$ $\times$ length $\times 0.5$.

(3) One-million ATF3-shRNA or Luc-shRNA transfected HCT116 cells were injected into the right lower liver lobe of mice to determine hepatic growth, as previously described [22]. Animals were monitored daily and sacrificed on day 28 ( $n=9-10 /$ group). Following necropsy, liver weight was measured and all tumor nodules counted and weighed.

(4) For testing peritoneal carcinomatosis, stable transfected HCT116 cells $\left(3 \times 10^{6}\right)$ were implanted into the abdominal cavity by intraperitoneal injection, as previously described [23]. Mice were monitored for 28 days and sacrificed; animals were evaluated for the presence of ascites and tumor nodules were counted.

\section{Immunohistochemical analysis}

Cryosections $(7 \mu \mathrm{m})$ and paraffin-embedded sections $(5 \mu \mathrm{m})$ were cut from xenograft tumors for immunohistochemical analyses. CD31-positive vessel area was analyzed by converting images to grey scale and setting a consistent threshold for all slides, as described [20,24]. Vessel area is expressed as pixels per high-power field [20].

\section{Human tissues}

For the analysis of ATF3 mRNA expression, snap frozen tissue samples of primary human colon carcinomas $(n=5)$ and corresponding non-neoplastic colon tissues $(n=5)$ were obtained from the anonymized tumor tissue bank of the Department of Pathology (University of Regensburg), as approved by clinical ethics committee [25]. Tumor characteristics were as follows: \#1 sigmoid colon: pT3, L0, V0, pN0, R0; \#2 cecum: pT4, pN2 (5/12), M1 (per), G2, R1, L1, V1; \#3 sigmoid colon: pT3, pN0 (0/15), G2, R0, L0, V0; \#4 cecum: pT3, pN2 (4/24), G3, L1, V0, R0; \#5 sigmoid colon: pT3, pN2, G2, R0, L1, V0. Patients did not receive neoadjuvant therapy or chemotherapy before surgery.

\section{Statistical Analyses}

Results from in vivo experiments were analyzed for significant outliers using Grubb's test http://www.graphpad.com. Tumor-associated variables in in vivo experiments were tested for statistical significance using the Mann-Whitney $U$ test for non-parametric data. The two-sided Student $t$ test was applied for analysis of in vitro data. All results are expressed as the mean \pm SEM.

\section{Results}

\section{Regulation and expression of ATF3 in cancer cells}

We previously observed that treatment of HCT116 and SW620 colon cancer cells with an Hsp90 inhibitor (17DMAG) substantially up-regulates constitutive ATF3 expression [8]. The biological effects of Hsp90 inhibitormediated induction of ATF3 are currently not known. To further validate these results, we investigated whether blocking Hsp90 also leads to ATF3 up-regulation in other human cancer cell types. Indeed we found that blocking Hsp90 induces ATF3 protein expression in human gastric (TMK-1), colon (HT29, HCT116, 


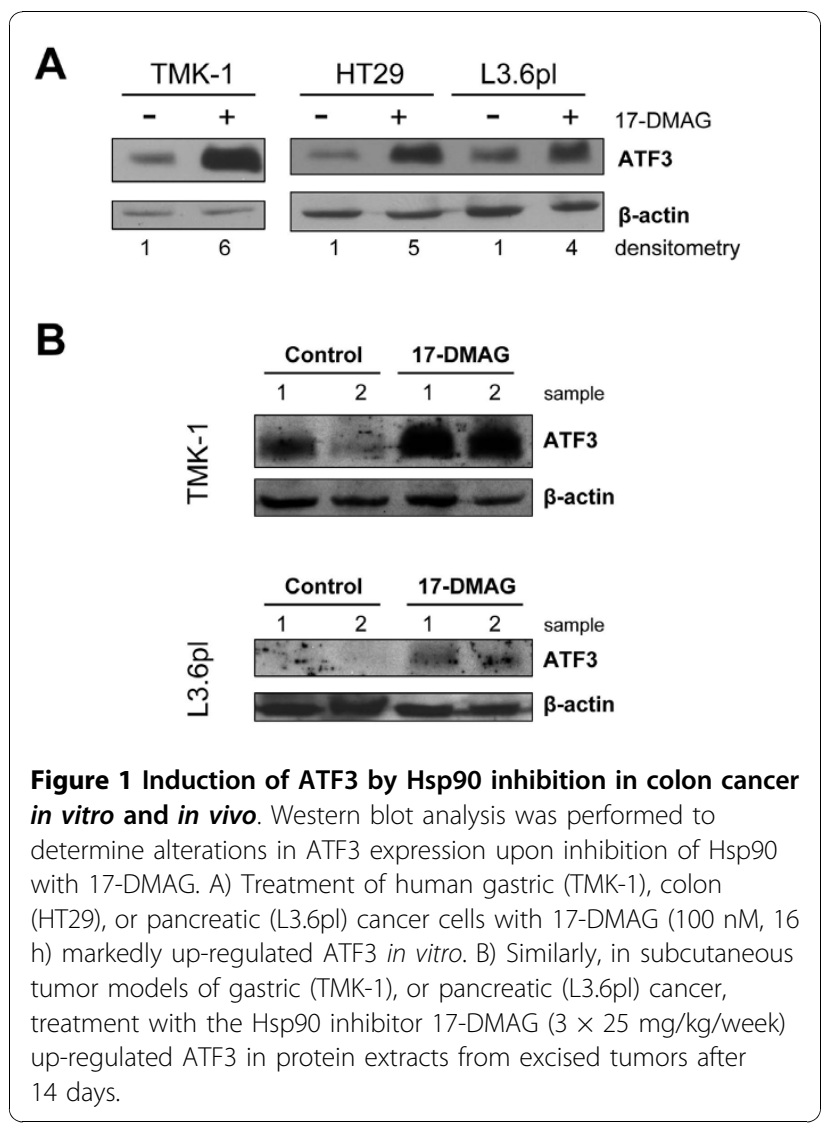

SW620), and pancreatic (L3.6pl) cancer cell lines (Figure $1 \mathrm{~A}$ and [8]). These results were validated in vivo using a model of subcutaneously implanted gastric (TMK-1), or pancreatic (L3.6pl) cancer cells where Hsp90 inhibitor treatment markedly induced ATF3 expression in respective tumors (Figure 1B).

Since blocking Hsp90 interferes with multiple cell signaling pathways, including MAPK/Erk, PI-3K/Akt, p38 and SAPK, we used in HCT116 cell line selective signaling inhibitors to determine the predominant signaling pathway involved in this Hsp90-inhibitor mediated ATF3 up-regulation (Figure 2). Inhibition of SAPK (SP600125) most robustly up-regulated ATF3 mRNA expression (Figure 2A). However, we additionally observed on a protein level that inhibition of either MAPK/Erk (UO126), or p38 (SB203580), could also up-regulate ATF3 expression in colon cancer cells (Figure 2B). We conclude from these experiments that ATF3 expression in colon cancer cells is complexly controlled through the interaction of multiple molecular signaling pathways. Because Hsp90 inhibition is known to affect a broad variety of signaling pathways, it is reasonable to conclude that inhibitors such as 17-DMAG overall lead to a net-gain in ATF3 expression.

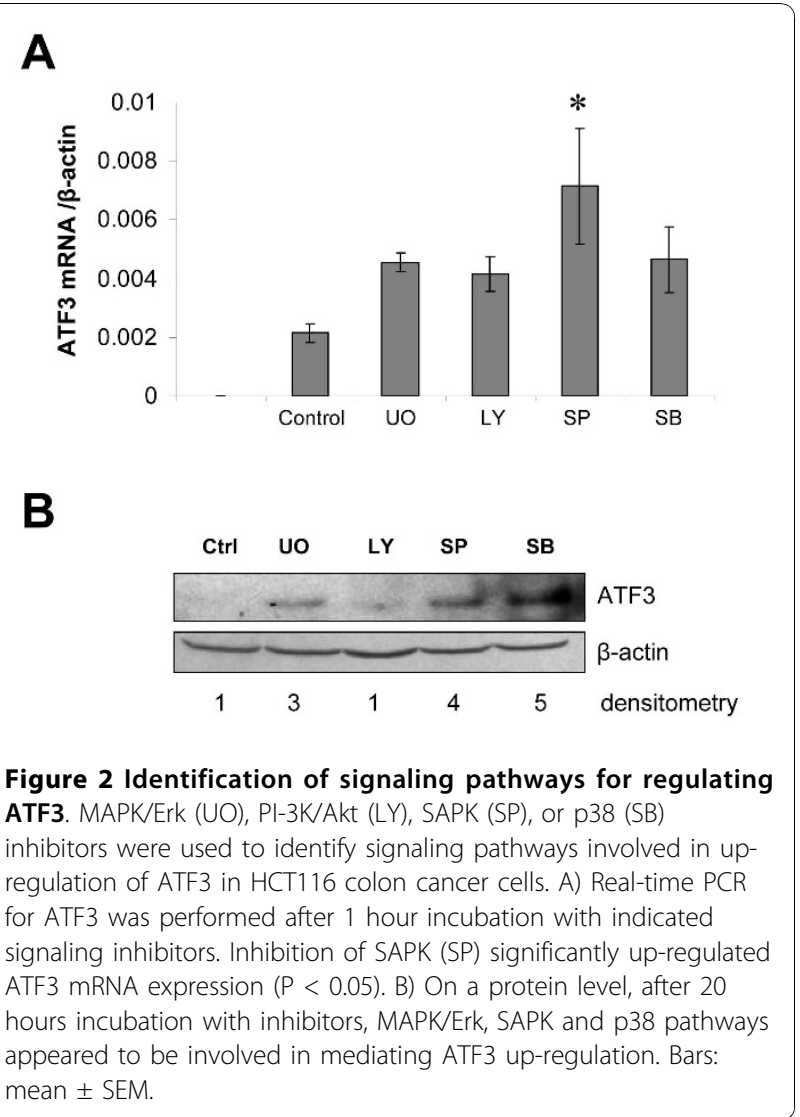

Effects of down-regulating ATF3 in colon cancer cells In view of the fact that ATF3 is stress-inducible and continuously detectable in colon cancer cells, we used an shRNA approach for specifically targeting ATF3 in HCT116 colon cancer cells, with the intention to determine the biological effects of a further ATF3 down-regulation in this cancer entity. Successful stable transfection with an ATF3-shRNA plasmid was verified by Western blotting and real-time PCR (Figure 3A, B). Importantly, down-regulation of ATF3 markedly increased the migration ability of colon cancer cells in vitro (Figure $3 \mathrm{C}$ ). Together, these in vitro experiments indicate that ATF3 down-regulation harbors the potential to increase the metastatic potential of colon cancer cells.

Impact of ATF3 down-regulation on tumor growth in vivo The effects of diminished ATF3 expression on tumor growth in vivo were first investigated in a subcutaneous tumor model using HCT116 cells. The results show that down-regulation of ATF3 by ATF3-shRNA leads to an increased tumor growth rate, as compared to Luc-shRNA transfected control cells (Figure 4A, B). Importantly, in vitro growth rates of Luc-shRNA and ATF3-shRNA transfected HCT116 cells were statistically 


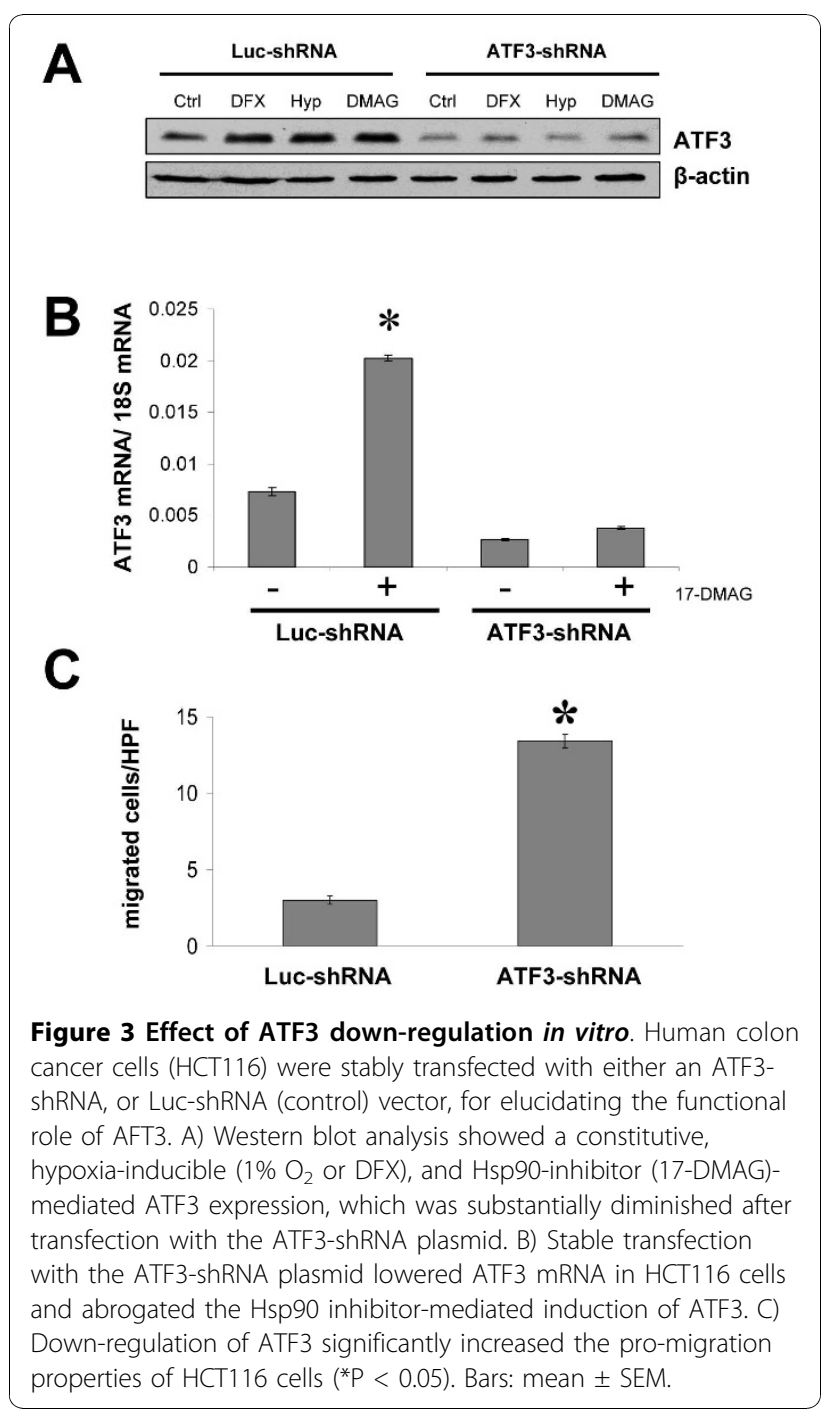

not different (data not shown). These in vivo results were confirmed by using one additional ATF3-shRNA transfected HCT116 clone (data not shown). Moreover, tumors from mice in the ATF3-shRNA group showed higher vascularisation in terms of an increased CD31positve vessel area (Figure 4C). We conclude from these experiments that ATF3 functions as a tumor suppressor and growth-inhibitory factor in HCT116 colon cancer.

\section{Impact of ATF3 down-regulation on colon cancer metastasis in vivo}

We next tested the effects of inhibited ATF3 expression on tumor metastasis in vivo in a model of hepatic tumor growth and in a model of peritoneal carcinomatosis. ATF3 silencing in HCT116 led to a substantial increase in hepatic tumor burden, as compared to Luc-shRNA transfected controls (Figure 5A, B). Furthermore, animals in the ATF3-shRNA group developed significantly more hepatic tumor nodules in liver lobes that had not been injected

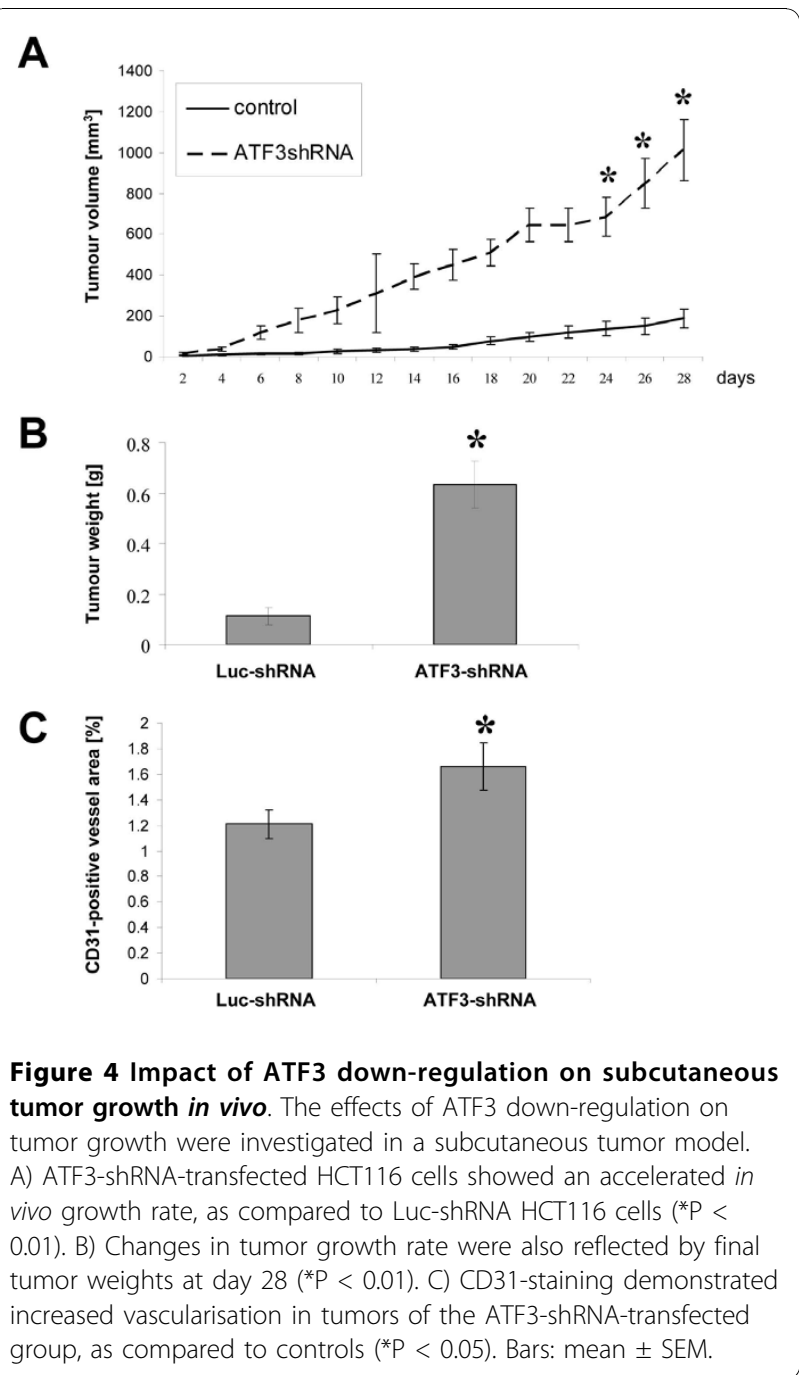

with tumor cells (Figure 5C). Similarly, in the peritoneal carcinomatosis model, animals in the ATF3-shRNA group developed multiple peritoneal nodules and $2 / 4$ (50\%) animals had detectable ascites (Figure 6A, B). These in vivo experiments support the hypothesis that ATF3 functions as a tumor suppressor and anti-metastatic factor in HCT116 colon cancer.

\section{Expression of ATF3 in human colon cancer specimens}

Since studies report conflicting results regarding the role and expression of ATF3 in colorectal cancers, we determined ATF3 mRNA expression in human colon cancer specimens. These results show that ATF3 is consistently expressed at exceptionally low levels in colon cancer tissues, as compared to corresponding normal tissues (Figure 7). We conclude that ATF3 is likely to be downregulated in colon cancers, hence supporting the rationale of therapeutically inducing ATF3 expression in this cancer entity. 


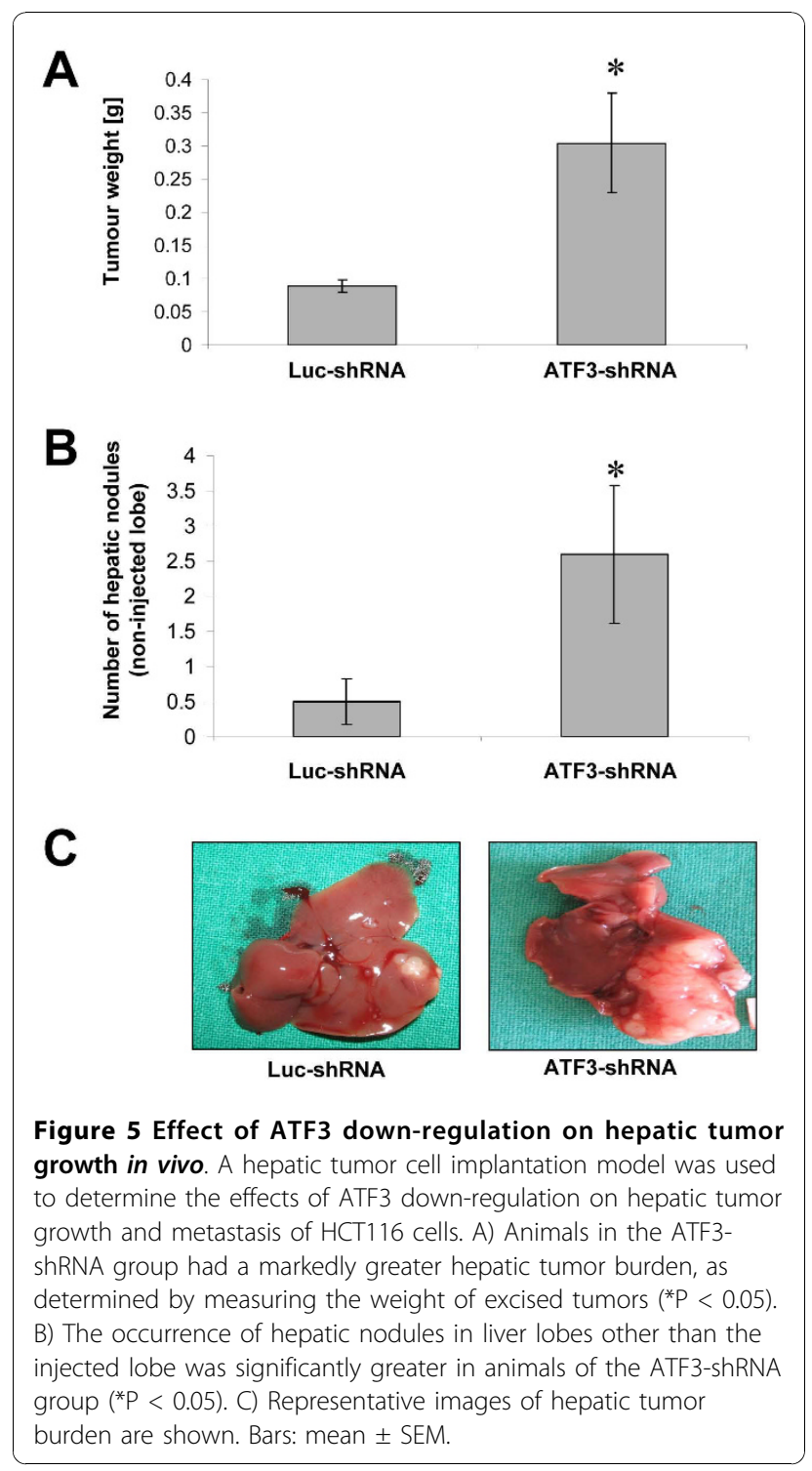

\section{Discussion}

Our recent observation that Hsp90 inhibition induces ATF3 in cancer cells and the lack of clarity regarding the biological effect of this transcription factor in oncology pressed our aim to define the role of ATF3 in colon cancer. We now have confirmed that blocking Hsp90 does indeed induce ATF3 in various cancer derived cell lines, including colon (HT29, HCT116), gastric (TMK1), and pancreatic $(\mathrm{L} 3.6 \mathrm{pl})$ cancer derived cells. Furthermore, this study is the first to demonstrate that loss of ATF3 via shRNA-mediated down-regulation increases the migration properties of HCT116 colon cancer cells in vitro and promotes tumor growth and metastasis in vivo. Hence, results from this study suggest that ATF3 functions as a tumor suppressor and anti-metastatic factor in HCT116 colon cancer, which is therapeutically inducible by blocking Hsp90.

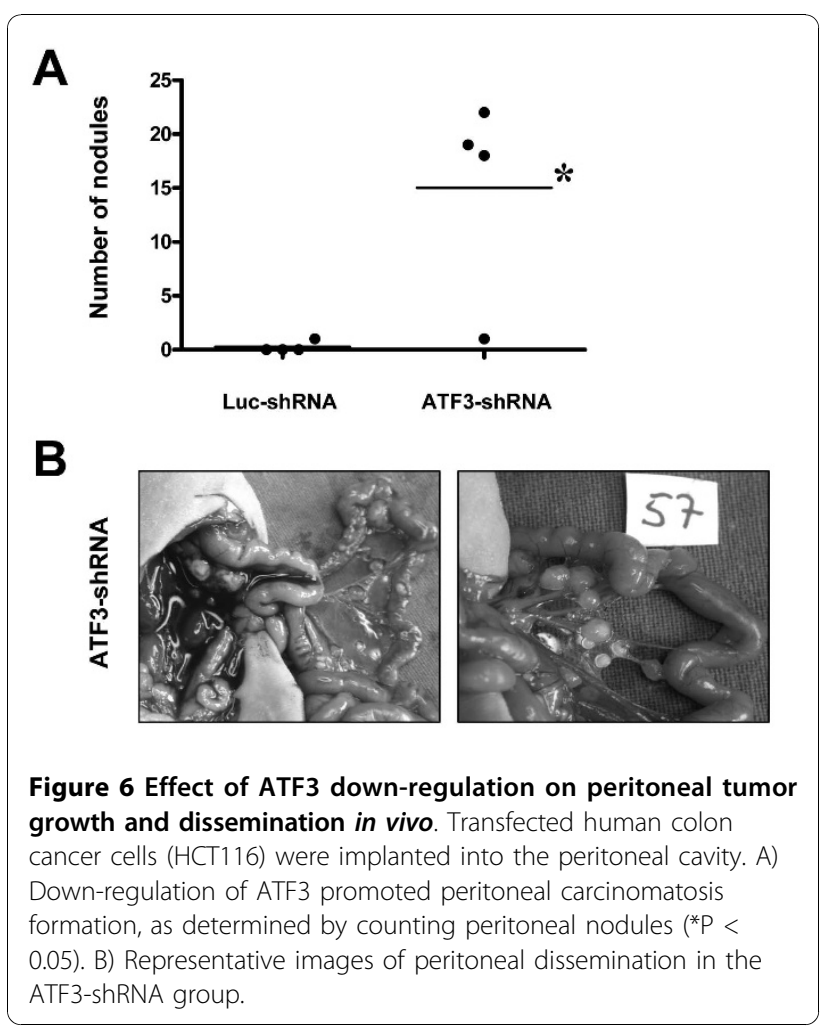

Recent publications have demonstrated a dichotomous role of ATF3. Depending on the cell type and malignancy, ATF3 can mediate either proliferative and pro-migration properties, or anti-proliferative and proapoptotic effects [26-29]. For instance, Yin and co-workers have demonstrated in in vitro experiments that ATF3 induces apoptosis in non-malignant mammary epithelial cells, but reduces apoptosis and enhances motility in breast cancer cells, suggesting an oncogenic role of ATF3 in breast cancer [15]. In colon cancer,

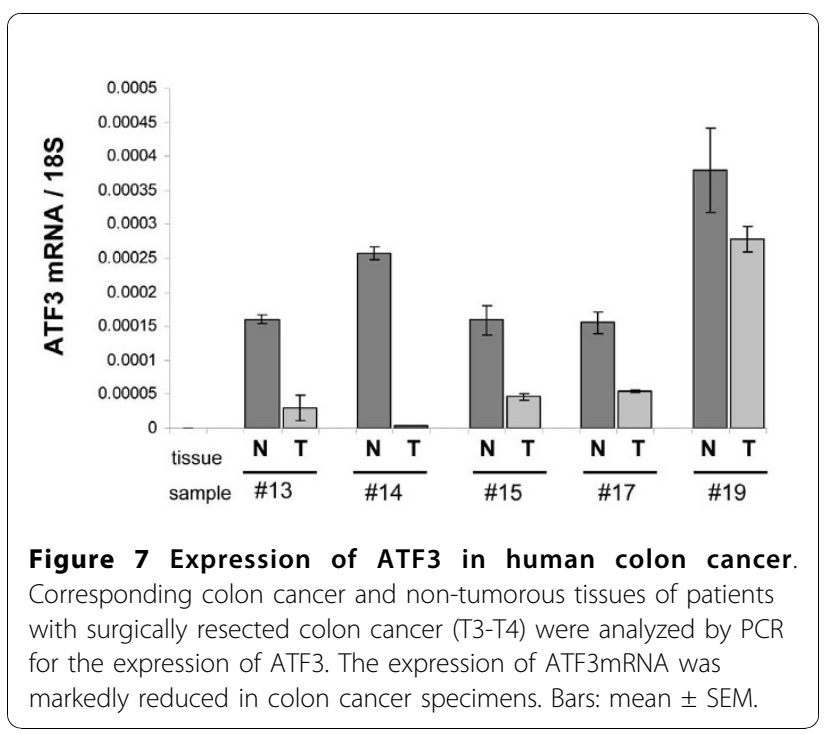


down-regulating ATF3 in HT29 colon cancer cells with antisense oligonucleotides apparently diminished entopic tumor growth and metastasis in mice [19]. In contrast, we could show that in HCT116 colon cancer, loss of ATF3 function does result in a higher pro-migration capacity in vitro and an accelerated tumor growth with increased metastasis in vivo. One explanation of this discrepancy might be the different genetic background of HT29 and HCT116 colon cancer cells. While HCT116 harbors mutant KRAS, HT29 colon cancer cells are wildtype for KRAS but harbor mutant BRAF. Recent publications have shown that the KRAS and BRAF mutation status of colon cancer cells influence the expression rates of multiple proliferative as well as apoptotic signaling intermediates (Kikuchi et al, Cancer Res 2009, Oliveira et al, Oncogene 2003, Seruca et al, 2009), including HIF1 $\alpha$ signaling and the MAPK/Erk and PI3K/Akt pathways which we identified as interacting with ATF3 (Figure 2). Furthermore, EGFR-targeting agents are clinically effective in the treatment of KRAS and BRAF wildtype tumors, whereas no clinical benefit could be proven for KRAS or BRAF mutant tumors (Lievre et al, Oncogene 2010). Thus, drug-induced overexpression of ATF3 may have beneficial effects in only a subset of colon cancer cells. This important result will be further addressed in future experiments, where loss of ATF3 function as well as ATF3-overexpression will be investigated in colon cancer cells with different genetic background.

In line with our findings in HCT116 colon cancer, tumor suppressive properties of ATF3 were suggested in a study by Oh et al., describing that ATF3 acts as tumorinhibiting factor in HeLa cervical cancer cells in vitro [30]. Moreover, Lu and co-workers elegantly demonstrated that ATF3 is capable of suppressing a Rasmediated tumorigenicity of murine fibroblasts (ATF3 ${ }^{-1-}$ versus $\mathrm{ATF}^{+/+}$fibroblasts) in an in vitro, as well as in an in vivo model, hence supporting our hypothesis of a tumor suppressive role. In conclusion, these discrepancies mirror the complex role of ATF3 which may not solely depend on the investigated cell line. The biological function of ATF3 in vivo may rather highly rely on the microenvironment of a defined tumor entity.

One clinical significance of our findings is that treatment-induced up-regulation of ATF3, as for example via Hsp90-inhibition or COX-2 inhibition, may be beneficial in some tumors for reducing growth and metastasis $[8,14]$. With respect to COX-2 inhibitors, experimental studies have nicely demonstrated that ATF3 may mediate anti-neoplastic and anti-invasive effects of such non-steroidal anti-inflammatory drugs [14]. In this study, overexpression of ATF3 inhibited invasion to a similar degree as sulindac sulfide treatment and antisense ATF3 increased invasion in vitro. This tumor suppressive effect of ATF3 is also supported by their findings, where transfection of cancer cells with a fulllength ATF3 vector suppressed tumorigenicity and invasiveness in vitro and tumor growth in vivo [14]. However, this group was not able to validate in an in vivo setting that loss of ATF3 function is conversely associated with increased growth rates and metastasis, hence our study further expands the knowledge on ATF3 function beyond these aspects. We observed an enhanced migration behavior after ATF3 inhibition in vitro and hypothesized that loss of ATF3 function may also lead to an increased tumor metastasis in vivo, an aspect that has not been comprehensively investigated to date. In subsequent hepatic and peritoneal tumor models, we were able to demonstrate a significant increase in tumor burden, cancer dissemination, and tumorigenicity upon further down-regulating ATF3. Thus, we propose that ATF3 functions as a tumor suppressor and anti-metastatic factor in HCT116 colon cancer. Moreover, in a recent publication, Ameri and colleagues could show that induction of ATF3 in hypoxic conditions, a common feature detectable in solid tumors, is independent of the transcription factor HIF-1 $\alpha$ [11]. The factors HIF- $1 \alpha$ and ATF3 are both induced by hypoxia and other cellular stressors, and both transcription factors regulate the expression of multiple genes during tumor progression and metastasis [11].

Importantly, and of high clinical relevance, we could show in the current and in one preliminary previous study that ATF3 expression can be induced in cancer cells by Hsp90 inhibition in vitro and in vivo. Inhibitors to Hsp90 are currently being investigated in a growing number of clinical trials http://www.clinicaltrials.gov. Thus, the present study not only adds an interesting new aspect to the multiple mechanisms of Hsp90-inhibition, but also provides reasonable evidence that an ATF3-induction by Hsp90 inhibition could be favorable for therapy of advanced colon cancer.

\section{Conclusion}

In conclusion, the present study shows that down-regulation of ATF3 enhances both invasive properties and tumor metastasis of HCT116 colon cancer cells in vivo. Our data suggest that induction of ATF3 may be valuable for improving therapy of colorectal cancer patients in terms of preventing hepatic and peritoneal metastasis. Furthermore, our study provides evidence that such ATF3-induction can be achieved by Hsp90-inhibition, which is particularly interesting since Hsp90-inhibitors are promising new agents for targeted therapy of advanced colorectal cancer and other malignancies [2].

\section{Abbreviation list}

17-DMAG: 17-(dimethylaminoethylamino)-17-demethoxygeldanamycin; ATF3: activating transcription factor-3; CREB: cyclic AMP response element binding 
protein; DMEM: Dulbecco's modified Eagle Medium; GLUT-1: glucosetransporter-1; HIF-1 $\alpha$ : hypoxia inducible factor-1alpha; HSF1: heat shock transcription factor 1; HSP70: heat shock protein 70; HSP90: heat shock protein 90; MAPK: mitogen-activated protein kinase; MTT-ASSAY: (3-(4,5Dimethylthiazol-2-yl)-2,5-diphenyltetrazolium bromide tetrazole assay; PBS phosphate buffered saline; PI-3K: phosphoinositide-3 kinase; SAPK: stressactivated protein kinase.

\section{Competing interests}

The authors declare that they have no competing interests.

\section{Authors' contributions}

$\mathrm{CH}$ - performed majority of experiments, adjusted study design and contributed to manuscript preparation; SAL - performed experiments and involved in study design; CM - performed experiments; AM - performed experiments and aided in animal study; SF - performed statistical analyses and helped with manuscript preparation; $\mathrm{CH}$ - collaborator for cell culture experiments; WD - provided human tissues from tissue bank and performed analyses of human specimens; HJS - manuscript editing and study refining; EKG - manuscript preparation and animal study supervision; OS - principal investigator, manuscript preparation, study design, animal study supervision. All authors read and approved the final manuscript

\section{Acknowledgements}

The authors thank Christine Wagner and Kathrin Stengel for excellent technical assistance.

These studies were supported in part by the AACR-Littlefield Grant for Metastatic Colon Cancer Research (American Association for Cancer Research, Philadelphia, PA) (O.S.), the German Cancer Aid (Deutsche Krebshilfe, Max-Eder Nachwuchsgruppen-Programm, Bonn, Germany) (O.S.), and a grant from the University of Regensburg, Medical Faculty (ReForM) (S. A.L.; C.M.)

\section{Author details}

${ }^{1}$ Department of Surgery, University of Regensburg Medical Center, FranzJosef Strauß Allee 11, 93042 Regensburg, Germany. 'Department of Internal Medicine I, University of Regensburg Medical Center, Franz-Josef Strauß Allee 11, 93042 Regensburg, Germany. ${ }^{3}$ Institute of Pathology, University of Regensburg Medical Center, Franz-Josef Strauß Allee 11, 93042 Regensburg, Germany. ${ }^{4}$ Departments of Hepatobiliary and Transplantation Surgery, University Medical Center Hamburg-Eppendorf, Martinistr. 52, 20246 Hamburg, Germany.

Received: 8 April 2010 Accepted: 3 December 2010 Published: 3 December 2010

\section{References}

1. Bagatell R, Whitesell L: Altered Hsp90 function in cancer: a unique therapeutic opportunity. Mol Cancer Ther 2004, 3(8):1021-1030.

2. Isaacs JS, Xu W, Neckers L: Heat shock protein 90 as a molecular target for cancer therapeutics. Cancer cell 2003, 3(3):213-217.

3. Nollen EA, Morimoto Rl: Chaperoning signaling pathways: molecular chaperones as stress-sensing 'heat shock' proteins. J Cell Sci 2002, 115(Pt 14):2809-2816.

4. Zhang H, Burrows F: Targeting multiple signal transduction pathways through inhibition of Hsp90. J Mol Med 2004, 82(8):488-499.

5. Gooljarsingh LT, Fernandes C, Yan K, Zhang H, Grooms M, Johanson K, Sinnamon RH, Kirkpatrick RB, Kerrigan J, Lewis T, et al: A biochemical rationale for the anticancer effects of $\mathrm{Hsp} 90$ inhibitors: slow, tight binding inhibition by geldanamycin and its analogues. Proc Natl Acad SCi USA 2006, 103(20):7625-7630.

6. Neckers L, Ivy SP: Heat shock protein 90. Curr Opin Oncol 2003, 15(6):419-424.

7. Taldone T, Gozman A, Maharaj R, Chiosis G: Targeting Hsp90: smallmolecule inhibitors and their clinical development. Curr Opin Pharmacol 2008, 8(4):370-374.

8. Moser C, Lang SA, Kainz S, Gaumann A, Fichtner-Feigl S, Koehl GE, Schlitt HJ, Geissler EK, Stoeltzing O: Blocking heat shock protein-90 inhibits the invasive properties and hepatic growth of human colon cancer cells and improves the efficacy of oxaliplatin in p53-deficient colon cancer tumors in vivo. Mol Cancer Ther 2007, 6(11):2868-2878.
9. Powers MV, Clarke PA, Workman P: Dual targeting of HSC70 and HSP72 inhibits HSP90 function and induces tumor-specific apoptosis. Cancer cell 2008, 14(3):250-262.

10. Powers MV, Clarke PA, Workman P: Death by chaperone: HSP90, HSP70 or both? Cell Cycle 2009, 8(4):518-526.

11. Ameri K, Hammond EM, Culmsee C, Raida M, Katschinski DM, Wenger RH, Wagner E, Davis RJ, Hai T, Denko N, et al: Induction of activating transcription factor 3 by anoxia is independent of p53 and the hypoxic HIF signalling pathway. Oncogene 2007, 26(2):284-289.

12. Lu D, Chen J, Hai T: The regulation of ATF3 gene expression by mitogenactivated protein kinases. Biochem J 2007, 401(2):559-567.

13. Lu D, Wolfgang CD, Hai T: Activating transcription factor 3, a stressinducible gene, suppresses Ras-stimulated tumorigenesis. J Biol Chem 2006, 281(15):10473-10481

14. Bottone FG, Moon Y, Kim JS, Alston-Mills B, Ishibashi M, Eling TE: The antiinvasive activity of cyclooxygenase inhibitors is regulated by the transcription factor ATF3 (activating transcription factor 3). Mol Cancer Ther 2005, 4(5):693-703.

15. Yin X, Dewille JW, Hai T: A potential dichotomous role of ATF3, an adaptive-response gene, in cancer development. Oncogene 2008, 27(15):2118-2127.

16. Wang A, Arantes S, Conti C, McArthur M, Aldaz CM, MacLeod MC: Epidermal hyperplasia and oral carcinoma in mice overexpressing the transcription factor ATF3 in basal epithelial cells. Mol Carcinog 2007, 46(6):476-487.

17. Bandyopadhyay S, Wang Y, Zhan R, Pai SK, Watabe M, liizumi M, Furuta E, Mohinta S, Liu W, Hirota S, et al: The tumor metastasis suppressor gene Drg-1 down-regulates the expression of activating transcription factor 3 in prostate cancer. Cancer Res 2006, 66(24):11983-11990.

18. Ishiguro $T$, Nagawa $H$ : ATF3 gene regulates cell form and migration potential of HT29 colon cancer cells. Oncol Res 2001, 12(8):343-346.

19. Ishiguro $T$, Nagawa $H$, Naito $M$, Tsuruo T: Inhibitory effect of ATF3 antisense oligonucleotide on ectopic growth of HT29 human colon cancer cells. Jpn J Cancer Res 2000, 91(8):833-836.

20. Lang SA, Moser C, Gaumann A, Klein D, Glockzin G, Popp FC, Dahlke MH, Piso P, Schlitt HJ, Geissler EK, et al: Targeting heat shock protein 90 in pancreatic cancer impairs insulin-like growth factor-I receptor signaling, disrupts an interleukin-6/signal-transducer and activator of transcription 3/hypoxia-inducible factor-1alpha autocrine loop, and reduces orthotopic tumor growth. Clin Cancer Res 2007, 13(21):6459-6468.

21. Bauer TW, Fan F, Liu W, Johnson M, Parikh NU, Parry GC, Callahan J, Mazar AP, Gallick GE, Ellis LM: Insulinlike growth factor-I-mediated migration and invasion of human colon carcinoma cells requires activation of c-Met and urokinase plasminogen activator receptor. Ann Surg 2005, 241(5):748-756, discussion 756-748

22. Stoeltzing O, Ahmad SA, Liu W, McCarty MF, Wey JS, Parikh AA, Fan F, Reinmuth N, Kawaguchi M, Bucana CD, et al: Angiopoietin-1 inhibits vascular permeability, angiogenesis, and growth of hepatic colon cancer tumors. Cancer Res 2003, 63(12):3370-3377.

23. Stoeltzing O, Ahmad SA, Liu W, McCarty MF, Parikh AA, Fan F, Reinmuth N, Bucana CD, Ellis LM: Angiopoietin-1 inhibits tumour growth and ascites formation in a murine model of peritoneal carcinomatosis. $\mathrm{Br} J$ Cancer 2002, 87(10):1182-1187.

24. Stoeltzing O, Liu W, Reinmuth N, Fan F, Parikh AA, Bucana CD, Evans DB, Semenza GL, Ellis LM: Regulation of hypoxia-inducible factor-1alpha, vascular endothelial growth factor, and angiogenesis by an insulin-like growth factor-I receptor autocrine loop in human pancreatic cancer. The American journal of pathology 2003, 163(3):1001-1011.

25. Dietmaier W, Gansbauer S, Beyser K, Renke B, Hartmann A, Rummele P, Jauch KW, Hofstadter F, Ruschoff J: Microsatellite instability in tumor and nonneoplastic colorectal cells from hereditary non-polyposis colorectal cancer and sporadic high microsatellite-instable tumor patients. Pathobiology 2000, 68(4-5):227-231.

26. Buganim $Y$, Kalo E, Brosh R, Besserglick H, Nachmany I, Rais Y, Stambolsky P, Tang $X$, Milyavsky M, Shats I, et al: Mutant p53 protects cells from 12-0tetradecanoylphorbol-13-acetate-induced death by attenuating activating transcription factor 3 induction. Cancer Res 2006 66(22):10750-10759.

27. Hartman MG, Lu D, Kim ML, Kociba GJ, Shukri T, Buteau J, Wang X, Frankel WL, Guttridge D, Prentki $M$, et al: Role for activating transcription 
factor 3 in stress-induced beta-cell apoptosis. Mol Cell Biol 2004, 24(13):5721-5732.

28. Hua B, Tamamori-Adachi M, Luo Y, Tamura K, Morioka M, Fukuda M, Tanaka Y, Kitajima S: A splice variant of stress response gene ATF3 counteracts NF-kappaB-dependent anti-apoptosis through inhibiting recruitment of CREB-binding protein/p300 coactivator. J Biol Chem 2006, 281(3):1620-1629.

29. Mashima T, Udagawa S, Tsuruo T: Involvement of transcriptional repressor ATF3 in acceleration of caspase protease activation during DNA damaging agent-induced apoptosis. J Cell Physiol 2001, 188(3):352-358.

30. Oh YK, Lee HJ, Jeong MH, Rhee M, Mo JW, Song EH, Lim JY, Choi KH, Jo I, Park Sl, et al: Role of activating transcription factor 3 on TAp73 stability and apoptosis in paclitaxel-treated cervical cancer cells. Mol Cancer Res 2008, 6(7):1232-1249

\section{Pre-publication history}

The pre-publication history for this paper can be accessed here: http://www.biomedcentral.com/1471-2407/10/668/prepub

doi:10.1186/1471-2407-10-668

Cite this article as: Hackl et al: Activating transcription factor-3 (ATF3)

functions as a tumor suppressor in colon cancer and is up-regulated upon heat-shock protein 90 (Hsp90) inhibition. BMC Cancer 2010 10:668.

\section{Submit your next manuscript to BioMed Central} and take full advantage of:

- Convenient online submission

- Thorough peer review

- No space constraints or color figure charges

- Immediate publication on acceptance

- Inclusion in PubMed, CAS, Scopus and Google Scholar

- Research which is freely available for redistribution

Submit your manuscript at www.biomedcentral.com/submit 